\title{
Prevalência de desmame precoce em um distrito sanitário da Amazônia Ocidental
}

Prevalence of early weaning in a sanitary district of Western Amazon

Rodrigo Pinheiro Silveira ${ }^{1}$

\section{RESUMO}

É bem conhecido o papel da amamentação na prevenção de diversos agravos à saúde. Este trabalho tem com o objetivo determinar a prevalência do desmame precoce na área de abrangência do Centro Estadual de Formação em Saúde da Família em Rio Branco, Acre. Trata-se de um estudo de prevalência, de base populacional, com todas as famílias que possuem crianças menores de 2 anos, de 6 bairros do Distrito Industrial de Rio Branco, utilizando como base o cadastramento do Sistema de Informação em Atenção Básica. Foi definido como desmame precoce a interrupção permanente da prática do aleitamento materno antes dos três meses de idade. A prevalência de desmame precoce encontrada foi de 16,1\% (63/392). Entre as crianças dos bairros de classe baixa, a prevalência de desmame precoce foi significativamente maior $(p<0,05)$ que nos bairros de classe média (19,6\% vs. 11,7\%). A mediana de amamentação foi de 12,2 meses. A prevalência de desmame antes dos três meses no Distrito do Tucumã é semelhante a de outros estudos realizados no Brasil e na região Norte.

\section{ABSTRACT}

It is well known how important are breastfeeding practices to prevent many health problems. The aim of this paper is to determine the prevalence of early weaning, in a district of Rio Branco, capital city of the State of Acre. This is a population-based prevalence study, involving all families with children behind two (2) years of age, in six (6) neighborhoods of the District of Tucumã. The population was defined based on the census provided by the Ministry of Health's Primary Attention Information System. Early weaning was defined as the total cessation of breastfeeding under three (3) months of age. The prevalence of early weaning was $16,1 \%$ (63/392). The prevalence among children from poorer neighbourhoods was significantly higher $(p<0.05)$ than among children from middle class neighbourhoods (19.6\% against $11.7 \%$ ). The medium duration of breastfeeding was 12.2 months. The prevalence of weaning found among children under three (3) months of age at the Tucumã District is similar to other studies in Brazil and in the North region.

PALAVRAS-CHAVE:

- Aleitamento Materno;

- Desmame;

- Epidemiologia;
KEY-WORDS:

- Breast feeding;

- Weaning;

- Epidemiology.

${ }^{1}$ Professor Assistente da Universidade Federal do Acre, Rio Branco, Brasil; Mestre em Medicina e Saúde pela UFBA. Residência em MFC pela UERJ. 


\section{I - Introdução}

A região Norte é uma das regiões mais carentes do Brasil, com ampla área territorial e baixa densidade populacional, apresentando diversas localidades de difícil acesso. Em 1999, a região apresentava uma das maiores taxas de mortalidade infantil do país, com 33,9 óbitos por 1.000 nascidos vivos, ficando atrás apenas da região Nordeste, com 52,4 óbitos por 1.000 nascidos vivos. Dentre os estados da região, o Acre é o que obteve o maior índice de mortalidade infantil, 44,2 por 1.000 nascidos vivos ${ }^{1}$.

Uma das estratégias para a redução da mortalidade infantil é o aleitamento materno. Inúmeros estudos vêm evidenciando os benefícios do aleitamento materno, tanto para o bebê quanto para as mães ${ }^{2,3,4,5,6}$. A declaração de Innocenti, de $1990^{7}$, apontou o incentivo ao aleitamento materno como ação que reduz a incidência e gravidade de doenças infecciosas, diminuindo a morbidade e a mortalidade infantil. Por esses motivos, a Organização Mundial de Saúde (OMS) preconiza o aleitamento materno exclusivo até os 6 meses de idade, e a extensão da prática da amamentação até 2 anos ou mais ${ }^{8}$.

No Brasil, em 1981, foi instalado o Programa Nacional de Incentivo ao Aleitamento Materno. $\mathrm{Na}$ década seguinte, algumas pesquisas, de âmbito nacional e regional, já mostravam aumento na duração mediana da amamentação 9,10,11,12.

Na região Norte são poucos os estudos que abordam a questão do aleitamento materno. Em 1999, o Ministério da Saúde, com base em estudos amostrais, estimou a prevalência de amamentação até os três meses no Brasil, em $83 \%$, sendo na região Norte de $88 \%$, e em Rio Branco de 81,2\%1.
Esta situação, e principalmente a falta de estudos sobre o tema na região, motivaram o presente estudo, que tem por objetivo determinar a prevalência do desmame precoce na área de abrangência do Centro Estadual de Formação em Saúde da Família em Rio Branco, bem como o de observar as diferenças entre os bairros mais carentes e os de melhor condição sócio-econômica ou ainda com e sem assistência pelas equipes de saúde da família.

\section{Metodologia}

O distrito sanitário do Tucumã faz parte do distrito industrial de Rio Branco, e se localiza na região periférica da cidade, distando aproximadamente $9 \mathrm{~km}$ do centro. O distrito é composto por 6 bairros: Conjunto Tucumã, Jardim Primavera, Mocinha Magalhães, Rui Lino, Joafra e Jardim Brasil. Estes bairros são assistidos pelo Centro Estadual de Formação de Pessoal em Saúde da Família (CEFSF), que conta com Policlínica de referência e 3 Unidades de Saúde da Família (USF) adstritas, implantadas no ano 2000.

As Unidades de Saúde da Família se localizam nos bairros Rui Lino, Jardim Primavera e Mocinha Magalhães. Nestas áreas as crianças menores de dois anos, inclusive as participantes da pesquisa, são assistidas pela Estratégia Saúde da Família (ESF). O bairro Conjunto Tucumã é desprovido de USF, sendo a população atendida na Policlínica e em clínicas e consultórios particulares da cidade de Rio Branco.

A população do distrito é de aproximadamente 12.500 habitantes. Os bairros são Heterogêneos, sendo o Conjunto Tucumã e o Rui Lino 
os de melhores condições sócio-econômicas, com habitações de alvenaria e ruas pavimentadas, além de serem assistidos pelas redes de água e esgoto da cidade. Os bairros Mocinha Magalhães, Jardim Primavera, Jardim Brasil e Joafra têm ruas sem pavimentação. Grande parte das casas é de madeira, utiliza água de poço, e os dejetos são despejados em fossas ou até em valas de esgoto a céu aberto. Esta diferença entre os bairros justificou a classificação para fins de comparação em "bairros de classe média", que são os dois primeiros e "bairros de classe baixa", representando os demais.

Este estudo faz parte de uma pesquisa mais abrangente, do tipo caso-controle, objetivando determinar fatores associados ao desmame precoce no Distrito Sanitário do Tucumã. Para tal, projetou-se a prevalência para a relação de casos e controles. O desmame precoce foi definido como a interrupção permanente da prática do aleitamento materno antes dos três meses de idade. Esta definição foi escolhida levando em consideração o risco de mortalidade nesta faixa etária e as medianas de amamentação das regiões do Brasil conforme estudos populacionais realizados nos últimos dez anos ${ }^{6,12,13}$. As demais definições seguem a classificação proposta pela $\mathrm{OMS}^{13}$ :

1. amamentação exclusiva: crianças que recebem somente leite materno e não ingerem nem mesmo água e/ou chás;

2. amamentação predominante: crianças que recebem leite materno e outros líquidos não-lácteos como água, chás, sucos, dentre outros;

3. amamentação: crianças que recebem leite materno, diretamente da mama ou ordenhado, independentemente de complementação.
A pesquisa se consistiu em um estudo populacional de prevalência, na modalidade de inquérito domiciliar, abrangendo a totalidade das crianças menores de 2 anos, residentes nos 6 bairros descritos, no período de julho a setembro de 2002. Foram excluídas do estudo as crianças que não viviam com a mãe biológica e as mães que não concordaram em participar do estudo.

O cadastramento contínuo das famílias dos bairros que possuem equipes de saúde da família foi a base para o inquérito nestas localidades. $O$ bairro Conjunto Tucumã foi cadastrado pelos estudantes de Medicina da Universidade Federal do Acre (UFAC), como parte da disciplina "Bases da Saúde da Família", realizado no mês de julho de 2002, resultando na realização do diagnóstico comunitário do bairro.

O instrumento de coleta foi um questionário simplificado, de fácil aplicação, baseado em trabalho realizado por Barros e Victora ${ }^{14}$. Este teve como objetivo saber se a criança iniciou a amamentação, se ainda estava mamando, se já havia ocorrido o desmame antes dos 3 meses de idade, a idade do desmame caso houvesse ocorrido. Foram também anotados a idade, o sexo e o local de moradia das crianças.

A análise estatística foi realizada utilizandose o teste do qui-quadrado para analisar a associação entre as características de interesse e o desfecho desmame precoce, sendo feita a estratificação no caso da comparação entre as classes sociais dos bairros do distrito sanitário que possuem ou não equipes de saúde da família. Como se trata de uma população e não uma amostra, já que o estudo se propôs a incluir todas as crianças menores de dois anos do distrito, o 
teste não se aplica para inferência estatística que mesmo assim, foi realizado para afastar a possibilidade de interferência do acaso nas diferenças encontradas, considerado a tamanho da população.

Para a determinação da duração mediana da amamentação e para a representação gráfica do desmame, foram utilizadas respectivamente, a tábua de vida e a curva de sobrevida (Kaplan-Meier). Para a comparação entre as curvas foi utilizado o teste de Log Rank.

Este projeto de pesquisa foi submetido ao Comitê de Ética em Pesquisa da Fundação Hospital Estadual do Acre (FUNDHACRE). Durante as visitas domiciliares para aplicação do questionário, foi explicitado o objetivo da pesquisa e foram feitas a leitura e a assinatura, quando de acordo, do Termo de Consentimento Livre e Esclarecido.

\section{Resultados}

Foram identificadas 417 crianças menores de 2 anos em todo o distrito sanitário, sendo a população total de aproximadamente 12.500 habitantes. Oito crianças $(1,9 \%)$ eram adotivas e foram excluídas da pesquisa, duas mães $(0,5 \%)$ não quiseram participar do estudo, e $15(3,6 \%)$ não foram encontradas nas diversas tentativas de visita. O total de perdas, adicionado às crianças excluídas da pesquisa, representou $6 \%(n=25)$ do total de crianças estudadas. A população estudada foi de 392 crianças menores de dois anos, residentes nos 6 bairros do distrito sanitário. As características da referida população estão descritas na tabela a seguir:
Tabela 1. Características da população estudada $(n=392)$.

\begin{tabular}{|lccc|}
\hline & $\mathrm{n}^{0}$ & $\%$ \\
\hline IDADE EM MESES (MÉDIA +/- DP) & 392 & 100 & $11,7+/-7,0$ \\
Masculino & 212 & 54,1 & $11,5+/-7,1$ \\
Feminino & 180 & 45,9 & $12,0+/-6,9$ \\
\hline TOTAL & 392 & 100 & \\
\hline
\end{tabular}

\begin{tabular}{|lcc|}
\hline LOCALDE RESIDÉNCIA & & \\
Conjunto Tucumã & 104 & 26,5 \\
Mocinha Magalhães & 130 & 33,2 \\
Rui Lino & 60 & 15,3 \\
Joafra & 18 & 4,6 \\
Jd. Brasil & 22 & 5,6 \\
Jd. Primavera & 58 & 14,8 \\
\hline TOTAL & 392 & 100 \\
\hline
\end{tabular}

\begin{tabular}{|c|c|c|}
\hline \multicolumn{3}{|c|}{ LOCAL DE RESIDÊNCIA, CONFRME A CLASSE SOCIAL } \\
\hline Baixa & 228 & 58,2 \\
\hline Média & 164 & 41,8 \\
\hline TOTAL & 392 & 100 \\
\hline
\end{tabular}

\begin{tabular}{|lcc|}
\hline \hline LOCAL DE RESIDÊNCIA, CONFORME PRESENÇA DE PSF \\
Com PSF & 288 & 73,5 \\
Sem PSF & 104 & 26,5 \\
\hline TOTAL & 392 & 100 \\
\hline
\end{tabular}

A proporção de crianças que iniciaram a amamentação foi de 98,5\% (Tabela 2). Esta proporção variou entre $97,1 \%$ no Conjunto Tucumã e $100 \%$ em Rui Lino, Joafra e Jardim Brasil. Não houve diferença estatística quanto ao início da amamentação entre os bairros que são cobertos por equipes de saúde da família (285/288) em comparação ao bairro desprovido desta equipe (101/104) (99,0\% vs. 97,1\%; $p=0,19)$.

À época do estudo, 219 crianças $(55,9 \%)$ estavam sendo amamentadas. A prevalência de desmame precoce foi de $16,1 \%$ (63/392), havendo diferença significativa entre os bairros estudados $\left(c^{2}=12,04 ; p<0,05 ; g . l .=5\right)$. A prevalência variou entre 10,8\%, no Conjunto Tucumã e 36,4\% no Jardim Brasil (Tabela 2). 
Tabela 2. Indicadores de amamentação no Distrito Sanitário do Tucumã em Rio Branco (Acre) n= 392.

\begin{tabular}{|lccc|}
\hline $\begin{array}{c}\text { Prevalência de } \\
\text { Bairros }\end{array}$ & $\begin{array}{c}\text { Proporção de } \\
\text { desmame precoce } \\
(\%)\end{array}$ & $\begin{array}{c}\text { Mediana da } \\
\text { crianças que } \\
\text { amamentaram (\%) }\end{array}$ & $\begin{array}{c}\text { amamentação } \\
\text { (meses) }\end{array}$ \\
\hline Conjunto Tacumã & 10,8 & 97,1 & 8,3 \\
Mocinha Magalhães & 14,8 & 98,5 & 19,2 \\
Rui Lino & 13,3 & 100,0 & 20,2 \\
Joafra & 27,8 & 100,0 & 7,9 \\
Jardim Brasil & 36,4 & 100,0 & 7,3 \\
Jardim Primavera & 21,1 & 98,3 & 12,5 \\
Total & 16,1 & 98,5 & 12,2 \\
\hline
\end{tabular}

Conforme descrito na Tabela 3, a prevalência de desmame precoce em crianças dos bairros de classe baixa foi significantemente maior que nos de classe média (19,6\% vs. 11,7\%; $\mathrm{p}<0,05)$.

Não houve diferença estatística quando se comparou a prevalência de desmame precoce saúde da família é de 100\%.

A duração mediana da amamentação foi de 12,2 meses. Esta variou entre 7,3 meses no Jardim Brasil e 20,2 meses no Rui Lino (Tabela 2). O Gráfico 1 (Curva de Kaplan-Meier) mostra o declínio progressivo da prática da amamentação durante os primeiros 24 meses de vida. As

Tabela 3. Indicadores de amamentação conforme a classe social e presença de Programa de Saúde da Família.

\begin{tabular}{|ccccc|}
\hline Bairros & $\begin{array}{c}\text { Prevalência de } \\
\text { desmame precoce } \\
\mathrm{n}(\%)\end{array}$ & $\begin{array}{c}\text { Razão de } \\
\text { prevalência } \\
\text { (IC 95\%) }\end{array}$ & $\begin{array}{c}\text { Proporção de } \\
\text { crianças que } \\
\text { amamentaram n (\%) }\end{array}$ & $\begin{array}{r}\text { Duração Mediana } \\
\text { da amamentação } \\
\text { (meses) }\end{array}$ \\
\hline $\begin{array}{l}\text { Classe Baixa } \\
\text { Classe Média }\end{array}$ & $\begin{array}{c}\text { (m }(19,6) \\
\text { 19 }(11,7)\end{array}$ & $1,67(1,0-2,7)$ & $225(98,7)$ & 13,2 \\
Com PSF & $52(18,2)$ & $1,69(0,9-3,1)$ & $285(99,0)$ & 9,6 \\
Sem PSF & $11(10,8)$ & & $101(97,1)$ & 14,3 \\
\hline
\end{tabular}

entre os bairros com e sem ESF (18,2\% vs 10,8\%; $\mathrm{p}=0,08)$. Esta mesma comparação, controlando o efeito da classe social do bairro, através de estratificação, mostrou redução da diferença de prevalências: nos bairros de classe média foi menor a diferença entre o que conta com a ESF (Rui Lino) e o Conj. Tucumã (13,3\% vs. 10,8\% - $p=0,63)$. Nos bairros de classe baixa, é impossível a comparação pois a cobertura com equipes de medianas dos bairros de classe baixa e média foram respectivamente 13,2 e 9,6 meses, alcançando diferença importante, mas sem significância estatística (Log Rank - p=0,64). Já a comparação das medianas dos bairros com e sem ESF mostrou grande diferença, tendo os bairros com ESF, mediana de 14,3 meses, e bairros sem ESF, mediana de 8,3. Esta diferença não atingiu significância $(p=0,09)$. 
As curvas de Kaplan-Meier sobre as comparações entre bairros de classe média e pobre e com ou sem ESF estão demonstradas nos gráficos 2 e 3.

Gráfico 1. Curva de duração da amamentação nos primeiros 24 meses de vida (Kaplan-Meier).

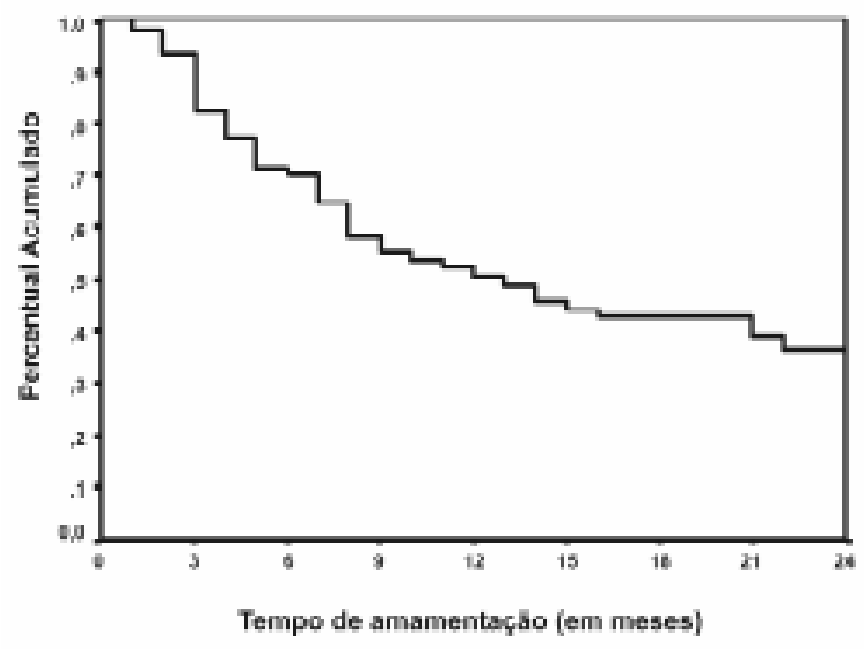

Gráfico 2. Curva de duração da amamentação por classe social dos bairros do Distrito Industrial de Rio Branco (Kaplan-Meier).

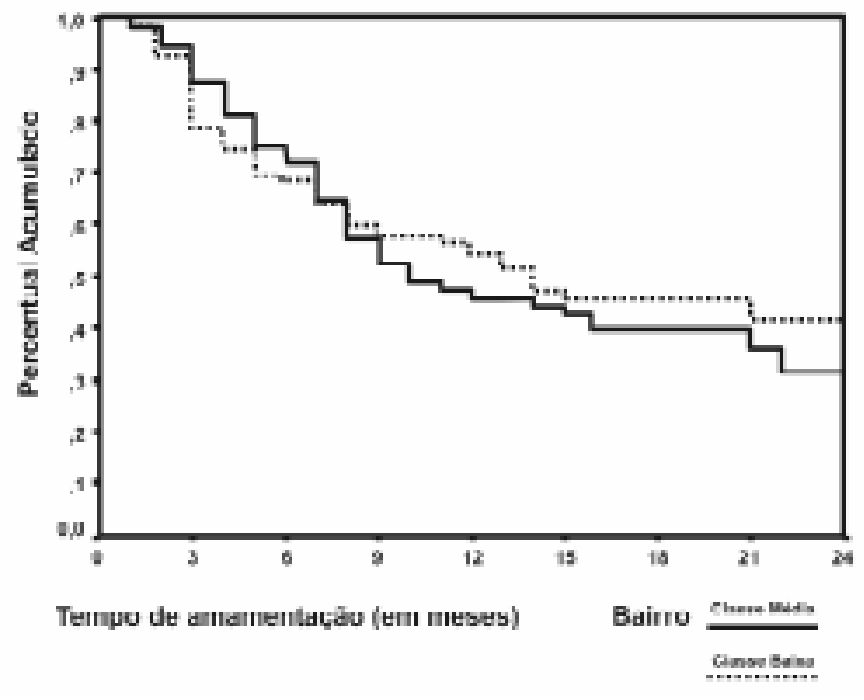

Gráfico 3. Curva de duração da amamentação por presença de equipe de PSF nos bairros do Distrito Industrial de Rio Branco (Kaplan-Meier).

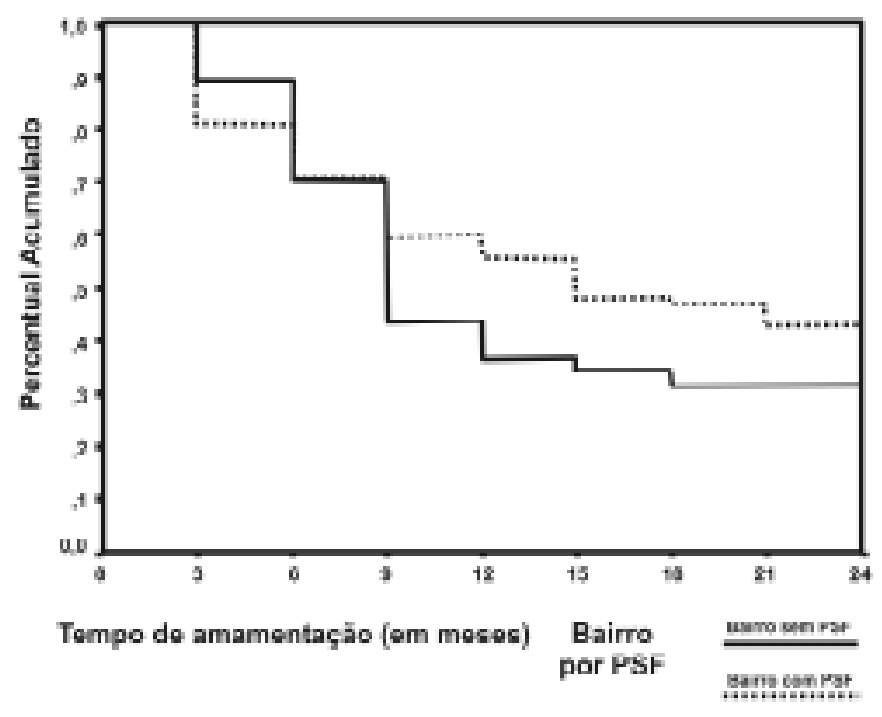

\section{Discussão}

A prevalência de desmame precoce observada no Distrito Sanitário do Tucumã $(16,1 \%)$ está em consonância com os últimos estudos realizados pelo Ministério da Saúde na região Norte, mais notadamente na cidade de Rio Branco. ${ }^{1}$ Em 1999, a prevalência de amamentação na cidade em crianças até os 3 meses foi $81,2 \%$, sendo $18,8 \%$ a prevalência de desmame precoce no terceiro mês de vida. Quando se comparam os resultados encontrados com outros estudos de âmbito nacional e regional, nota-se que a prevalência de crianças com desmame precoce $(16,1 \%)$ no Distrito do Tucumã é relativamente baixa $^{10,11,15,16,17,18,19}$.

A proporção de crianças que iniciaram a amamentação no Distrito foi de $98,5 \%$. Esta foi superior aos encontrados na maioria dos estudos analisados ${ }^{10,11,15,17,18,20}$. Nos estudos de âmbito nacional, foram encontradas em 1986, 1989 e 
1996, proporções de crianças que iniciaram a amamentação de $86 \%, 97,2 \%$ e $92 \%$, respectivamente. ${ }^{10,17}$ Em 96 a região Norte obteve 95,4\%, o melhor índice entre as regiões do País. ${ }^{17} \mathrm{Em}$ estudos realizados nas regiões Sul e Sudeste, esta proporção variou entre $94 \%$ e $98 \%{ }^{11,15,18,20}$. A maior proporção encontrada no Brasil foi no estudo de Marques et al. ${ }^{19}$ em Pernambuco: 99\%.

Estudos que trabalham com indicadores de aleitamento materno mostram grande variabilidade entre os diferentes continentes, países ou regiões, refletindo possivelmente a existência de crenças e costumes distintos. No continente africano, virtualmente todas as crianças são amamentadas no mínimo até 1 ano de vida, chegando a mediana de amamentação a

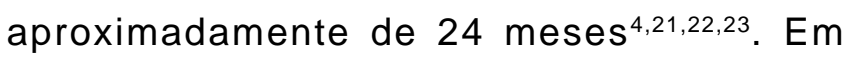
contrapartida, dados dos Estados Unidos indicam que pouco mais da me- tade das crianças do país são amamentadas ${ }^{24,25}$.

Entre os países da América Latina há, basicamente, dois padrões: um com marcada redução da prevalência da amamentação durante o primeiro ano de vida, no qual se incluem Brasil, México, Colômbia, República Dominicana e Trinidad e Tobago, e outro grupo formado por Guatemala, Bolívia, Equador e Peru, com redução menos pronunciada no primeiro ano e amamentação mais duradoura ${ }^{26}$. No Brasil, as regiões Sul, Sudeste, Centro-Oeste e Nordeste apresentam padrão semelhante, enquanto a região Norte apresenta baixas medianas de amamentação exclusiva, mas altas medianas de amamentação ${ }^{10,17}$.

A duração mediana da amamentação no distrito sanitário do Tucumã foi alta $(12,2$ meses), especialmente ao se comparar com estudos realizados em outras regiões do País. Em 1996, a mediana foi de 10,3 meses na região Norte ${ }^{17} \mathrm{e}$, na mesma época, variou de quatro a seis meses nas regiões Sul e Sudeste ${ }^{11,15,18,20}$.

No presente estudo, houve diferença significativa de prevalência de desmame precoce entre os bairros do distrito sanitário estudado. Os bairros de classe média apresentaram as menores prevalências, enquanto os bairros de classe baixa evidenciaram as maiores. Este resultado é socialmente relevante visto que o desmame precoce pode levar a uma série de problemas de saúde, e incide principalmente nas classes mais baixas, onde as dificuldades para a manutenção da saúde são maiores, o que aumenta mais ainda o risco de morbidade e mortalidade nos primeiros anos de vida. Não obstante, na análise da curva de Kaplan-Meier que compara os bairros de classes média e baixa, pode-se notar que o desmame nos primeiros meses é mais pronunciado nos bairros de classe baixa, levando a uma maior prevalência de desmame precoce, porém a manutenção da amamentação ao longo dos dois primeiros anos é mais duradoura nesses bairros, gerando mediana de amamentação maior.

As diferenças de prevalência e duração de amamentação entre mães de condições sócioeconômicas também foram descritas por outros autores. Victora e colaboradores ${ }^{27}$ demonstraram que quanto menor a renda familiar, menor a duração da amamentação, fato confirmado por outros estudos no Brasil ${ }^{10,15,28,29}$. 
O padrão da curva de amamentação entre classes sociais, com queda mais acentuada da amamentação nos primeiros meses e maior duração na classe mais baixa, também foi evidenciada em dois estudos de coorte realizados em Pelotas, Rio Grande do Sul ${ }^{11}$. Nestes estudos, a variável que expressa a classe social foi renda familiar. No estudo de âmbito nacional de 1989 este fato não ocorreu, ficando a curva de amamentação na classe mais baixa sempre inferior às curvas das classes média e alta ${ }^{10}$.

As práticas em aleitamento materno da região Norte caracterizam-se pela duração mais prolongada da amamentação, mas com a introdução precoce de outros alimentos, tais como chás mingaus de banana ou aipim, e leite em pó, padrão apresentado freqüentemente pelas famílias mais carentes. ${ }^{10}$ Deste modo, o padrão regional é de mediana de amamentação relativamente prolongada associada a baixas prevalências de amamentação exclusiva, o que aumenta bastante o risco de doenças infecto-parasitárias como diarréias e infecções respiratórias agudas.

Kitoko et al. ${ }^{30}$, ao comparar os indivíduos em duas cidades de regiões distintas do Brasil e padrões opostos de condição sócio-econômica, em Florianópolis e João Pessoa, verificaram parâmetros de melhor qualidade em Florianópolis, com maiores durações de amamentação e de amamentação exclusiva e menor proporção de uso de mamadeiras.

As diferenças de prevalência de desmame precoce encontradas em bairros que têm ou não a ESF, $18,2 \%$ vs. $10,8 \%$, provavelmente são influenciadas pela classe social, pois todos os bairros de menor condição no distrito são cobertos por estas equipes. Esta hipótese se torna mais provável quando se compara as prevalências do Conjunto Tucumã e do Rui Lino (10,8\% e 13,3\%). Ambos são bairros de classe média, mas apenas o segundo possui a ESF. A diferença entre as prevalências cai sensivelmente em relação à primeira comparação.

Em contrapartida, a comparação das medianas de amamentação entre os bairros com e sem a ESF mostra duração maior nos bairros que possuem a ESF (14,3 vs. 8,3 meses), embora não alcançada significância estatística $(p=0,09)$.

Por ser parte de de um processo de pesquisa em andamento, estudo caso-controle, outras variáveis pesquisadas não foram incluídas no presente estudo, impossibilitando inclusive a aplicação de análise multivariada. A despeito das limitações metodológicas, o estudo e seus resultados são de extrema validade para as equipes de saúde que trabalham na região, pois servem de base para o planejamento das ações de saúde na promoção do aleitamento materno e na prevenção da mortalidade infantil. Os resultados descritos também colocam em evidência a eqüidade, um princípios básico do Sistema Único de Saúde, quando mostra a diferença da prevalência de desmame entre os bairros estudados.

Apesar das diferenças encontradas entre os bairros do Distrito do Tucumã em Rio Branco, faz-se necessário um estudo de metodologia apropriada para avaliar a relação entre as condições de vida e os indicadores de aleitamento.

A pesquisa suscita algumas perguntas que poderiam ser respondidas com estudos posteriores. O estudo caso-controle possibilitaria o meIhor entendimento sobre os fatores associados ao 
desmame precoce no distrito do Tucumã. Posteriormente há que se ter noção das causas imediatas que levam ao desmame precoce, contribuindo ainda mais para as ações de saúde na região.

\section{Referências Bibliográficas}

1. Brasil, Ministério da Saúde. Rede Interagencial de Informações para a Saúde, IDB. 2001. Disponível em URL: http://tabnet.datasus.gov.br/cgi/ idb2001.htm [2002 Nov 18]

2. Giugliani ERJ. Amamentação: como e porque promover. Jornal de Pediatria 1994; 70: 38-51.

3. Newcomb PA, Storer BE, Longnecker MP, Mittendorf R, Greenberg ER, Clapp R, et al. Lactation and a reduced risk of premenopausal breast cancer. New England Journal of Medicine 1994; 330: 81-87.

4. WHO Collaborative Study Team on the Role of Breastfeeding on the Prevention of Infant Mortality. Effect of Breastfeeding on infant and child mortality due to infectious diseases in less develop countries: a pooled analysis. Lancet 2000; 355: 451-455.

5. Acog Educational Bulletin. Breastfeeding: Maternal and Infant Aspects. International Journal of Gynecology \& Obstetrics 2001, 74: 217-232.

6. Betrán AP, Onís M, Lauer JA, Villar J. Ecological study of effect of breast feeding on infant mortality in Latin America. British Medical Journal 2001, 323: 303-306.

7. WHO (World Health Organization)/UNICEF. Innoceti declaration on protection, promotion and support of breastfeeding. Ecological Food Nutrition 1991, 26: 271-273.

8. WHO (World Health Organization). The Optimal Duration of Exclusive Breastfeeding a Sistematical
Review. WHO; 2002.

9. Rea MF, Berquó ES. Impact of the Brazilian national breast-feeding programme on mothers in greater Sao Paulo. Bulletin of World Health Organization 1990, 68: 265-271.

10. Leão MM, Coitinho DC, Recine E, Costa LAL, Lacerda AJO. Perfil do aleitamento materno no Brasil. In: Fundação IBGE, Perfil estatístico de crianças e mães no Brasil. Rio de Janeiro; 1992. p. 97-109.

11. Horta BL, Olinto MTA, Victora CG, Barros FC, Guimarães PRV. Amamentação e padrões alimentares em crianças de duas coortes de base populacional no Sul do Brasil: tendências e diferenciais. Cadernos de Saúde Pública 1996; 12 (suppl.1): 43-48.

12. Lamounier JA. Tendências em Aleitamento Materno no Brasil. Revista Médica de Minas Gerais 1999, 9: 59-65.

13. WHO (World Health Organization). Programme for Control of Diarrhoeal Diseases. Indicators for assessing breastfeeding practices: update, 10. Geneva: WHO; 1992.

14. Barros FC, Victora CG. Epidemiologia da Saúde Infantil: Um Manual para Diagnósticos Comunitários. São Paulo: HUCITEC-UNICEF; 1991.

15. Carvalhães MABL, Parada CMGL, Manoel CM, Venâncio SY. Diagnóstico da situação do aleitamento materno em área urbana do Sudeste do Brasil: utilização de metodologia simplificada. Revista de Saúde Pública 1998, 32: 430-436.

16. Vieira GO, Glisser M, Araújo SPT, Sales NA. Indicadores do aleitamento materno na cidade de Feira de Santana, Bahia. Jornal de Pediatria 1998, 74: 11-16. 
17. Bemfam. Pesquisa Nacional Sobre Demografia e Saúde: Amamentação e Situação Nutricional das Mães e Crianças. Rio de Janeiro: 2ª edição; 1999. p.125-30.

18. Passos MC, Lamounier JÁ, Mariano da Silva CA, Freitas SN, Baudson MFR. Práticas de amamentação no município de Ouro Preto, MG, Brasil. Revista de Saúde Pública 2000, 6: 617-622. 19. Marques NM, Lira PIC, Lima MC, Silva NL, Filho MB, Huttly SRA, et al. 2001. Breastfeeding and Early Weaning Prectices in Northeast Brazil: A Longitudinal Study. Pediatrics 1999, 108: E66.

20. Weiderpass E, Barros FC, Victora CG, Tomasi $E E$, Halpern R. Incidência e duração da amamentação conforme tipo de parto: estudo longitudinal no Sul do Brasil. Revista de Saúde Pública 1998, 3: 225-231.

21. Jakobsen MS, Sodemann M, Molbak K, Aaby P. Reason for termination of breastfeeding and the lenth of breastfeeding. International Journal of Epidemiology 1996, 25: 115-121.

22. Chinebuah B, Perez-Escamilla R. Unplanned pregnancies are associated with less likelihood of prolonged breast-feeding among primiparous women in Ghana. Journal of Nutrition 2001, 131 : 1247-1249.

23. Simondon KB, Costes R, Delaunay V, Diallo A, Simondon F. Children's height, health and appetite influence mothers' weaning decisions in rural Senegal. International Journal of Epidemiology 2001, 30: 476-481.

24. Erten OI, Votto N, Leventhal J.M. The Timing and Predictors of the Early Termination of Breastfeeding. Pediatrics 2001, 107: 543-548.

25. Arora S, Mcjunkin C, Wehrer J, Kuhn P. Major Factors Influencing Breastfeeding Rates: Mothers
Perception of Father's Attitude and Milk Suply. Pediatrics 2000, 106: E67.

26. Perez-Escamilla R. Breastfeeding paterns in nine Latin American and Caribbean countries. Bulletin of Panamerican Health Organization 1993, 27: $32-42$.

27. Victora CG, Barros FC, Vaughan JP. Epidemiologia da Desigualdade. São Paulo: HUCITEC-UNICEF; 1988.

28. Rea MF, Venâncio SI, Batista LE, Santos RG, Greiner T. Possibilidades e limitações da amamentação entre mulheres trabalhadoras formais. Revista de Saúde Pública 1997, 2: 402416.

29. Kummer SC, Giugliani ERJ, Susin LO, Folletto JL, Lermen NR, Wu VYJ, et al. Evolução do padrão de aleitamento materno. Revista de Saúde Pública 2000. 34: 143-148.

30. Kitoko PM, Réa MF, Venancio SI, Vasconcelos ACCP, Santos EKA, Monteiro CA. Situação do aleitamento materno em duas capitais brasileiras: uma análise comparada. Cadernos de Saúde Pública 2000, 16: 1111-1119

\section{Endereço para correspondência:}

Rodrigo Pinheiro Silveira

Rua do Seringueiro, 282 - Vila Acre

Rio Branco AC - CEP.: 69900-000

Endereço Eletrônico: ropsilv@uol.com.br 